\title{
Relectura de la educación por competencias desde el pragmatismo de John Dewey
}

\section{Rereading competency-based learning from John Dewey's pragmatism}

\author{
Gonzalo Jover \\ Grupo de Investigación sobre Cultura \\ Cívica y Políticas Educativas \\ Universidad Complutense \\ gjover@ucm.es \\ Fecha de recepción: 11-1-2015 \\ Fecha de revisión: 2-3-2015
}

\author{
Alicia García Fernández \\ Grupo de Investigación sobre Cultura \\ Cívica y Políticas Educativas \\ Universidad Complutense \\ aligar07@ucm.es
}

Fecha de aceptación: 20-3-2015

Fecha de publicación: 27-3-2015

\section{Palabras clave:}

Democracia; aprendizaje por competencias;

pragmatismo; política educativa.

\section{Resumen}

En las últimas décadas se ha generado un interés renovado por el pragmatismo y John Dewey. En el ámbito de la educación, la apelación a Dewey se ha convertido en un recurso habitual como antecedente de diversas propuestas, entre ellas, el aprendizaje por competencias. Emanado del ámbito de la formación y la gestión profesional, la necesidad de adaptar los sistemas de educación y formación a las demandas planteadas por la sociedad y la economía actual, ha hecho que este enfoque del trabajo pedagógico se haya instalado con fuerza en las políticas educativas nacionales e internacionales. El objetivo en este artículo es mostrar las posibilidades de filiación que existen entre el aprendizaje por competencias y las propuestas del filósofo y pedagogo americano. Para ello, en primer lugar se rastrea esta filiación en el marco del empeño deweyano de reconstrucción democrática, mediante la exhortación a un nuevo individualismo que lograse
Keywords:

Democracy; competency-based learning; pragmatism; educational politics.

\section{Abstract}

Over the last decades, a renewed interest in pragmatism and John Dewey thought has emerged. In the field of the Education, the reference to Dewey has become a common resource as background for various proposals, including the model of learning based on competencies. Emanated from the field of professional management and training, the need to adapt education to the requirements of the society and economy, has put this approach into the heart of national and international educational policies. In this paper we attempt to show the possibilities of filiation that exist between competencybased learning and the ideas of the American philosopher and educator. First, we focused on Dewey democracy reconstruction through the appeal to a new individualism which conciliates the development of the individuality with the participation in the spirit of a community life. Then the analysis is moved to Dewey's criticism to dualisms 
conciliar el desarrollo de la individualidad con la participación en un espíritu de vida comunitario. A continuación, el análisis se mueve al rechazo de Dewey a los dualismos y los debates que mantuvo con respecto a la articulación de la formación profesional y la educación general. Por último, se exploran las consecuencias que para el enfoque de las competencias cabe extraer de la propuesta de cambio de una lógica absolutista a una lógica de la experimentación. La filosofía de la educación de Dewey no necesariamente es contraria a una formación en competencias. Él insistió mucho en la necesidad de aproximar la educación a las necesidades sociales del momento, sin dejarse atrapar por la antigua distinción que rebaja la educación profesional y práctica a una formación de segunda categoría. Pero habría sido reacio a una formación en competencias según una lista cerrada y preestablecida de antemano, que prefijase un determinado perfil de la persona a alcanzar, obstruyendo la libertad y creatividad del alumno.

\section{Introducción}

En las últimas décadas se ha generado un interés renovado por el pragmatismo. La antaño denostada filosofía pragmática ha logrado volver a abrirse un espacio destacado en el panorama filosófico internacional, como ponen de manifiesto las recurrentes apelaciones tanto a los pragmatistas clásicos como a los neopragmatistas contemporáneos. Dentro de este redescubrimiento, las referencias a John Dewey resultan cada vez más frecuentes. Atrás quedan los tiempos -las décadas que siguieron a la Segunda Guerra Mundial- en que el pensamiento de Dewey parecía condenado al ostracismo, arrinconado en el mundo intelectual norteamericano de postguerra por el auge del positivismo lógico y la filosofía analítica. Diversos factores han colaborado a esta revitalización, entre ellos cabe subrayar, con Morán, el atractivo que hoy suscita la figura de Dewey como intelectual público, preocupado en su tiempo por unos problemas que en buena parte siguen siendo también los del nuestro (Morán, 2009, pp. 11$16)$. and the discussion around vocational training and general education. To conclude, we explore the consequences of the change from an absolutistic logic to an experimental one on this model of learning. Dewey's philosophy of education is not necessary contrary to the competency-based model. He insisted in the necessity to connect education within society and rejected understanding vocational training as a second-class education. However, he would have been reluctant to an education built over a closed list of competencies determining the profile of the person to achieve, and obstructing the freedom and creativity of the student

En el ámbito concreto de la educación, la apelación a Dewey se ha convertido en un recurso habitual como antecedente justificativo de las más variadas propuestas, desde la práctica reflexiva en la formación y comprensión del trabajo de los profesores (Rodgers, 2002) a las experiencias de aprendizaje en servicio (Giles \& Eyler, 1994; You \& Rud, 2010) por poner dos ejemplos. Otro de los aspectos de las actuales reformas educativas en el que se ha hecho frecuente acudir a Dewey, es el del aprendizaje por competencias. McCowan (1998) por ejemplo, lo considera uno de los inspiradores tempranos de este enfoque a comienzos del siglo XX, junto con el padre de la organización científica del trabajo, Frederick W. Taylor y el psicólogo precursor del conductismo Edward L. Thorndike, con quien Dewey compartió no solo la ubicación en Columbia University durante varias décadas, sino también el interés por la educación progresista y la confianza en la capacidad de la ciencia para mejorar la educación (Koschmann, 2000). 
Pedagógicamente, el interés por Dewey ya no es, pues, solo, ni prioritariamente, histórico, sino que se produce asociado a ciertos temas de "irrésistible ascension". Con esta expresión definió Marc Romainville a mediados de los años noventa la emergencia de la noción de competencia como eje de las políticas curriculares (Romainville, 1996). Esta adquirió inicialmente relevancia en el campo educativo en relación con la enseñanza y el aprendizaje de las lenguas en políticas desarrolladas por el Consejo de Europa, preludio del establecimiento del Marco Común de Referencia de las Lenguas (MCER) que acogía el concepto la "competencia comunicativa" introducido por Hymes. Su uso se popularizó en la década de los ochenta y se extendió en los noventa. En 1997 se aprobaba en Bélgica el decreto Missions, que establecía una reforma educativa centrada en las competencias. Ese mismo año, la OCDE lanzaba el Programa para la Evaluación Internacional para Estudiantes (conocido como PISA, por sus siglas en inglés) con la finalidad de evaluar la adquisición por los estudiantes, al término de la escolaridad obligatoria, de conocimientos y destrezas en las áreas de lectura, matemáticas y resolución de problemas. Paralelamente, la OCDE, con el apoyo de la Oficina de Estadística Federal de Suiza, promovía el Proyecto DeSeCo, que tenía el objetivo de definir un conjunto de competencias que sirviese de referencia internacional a las políticas educativas, y proporcionase un marco que guiase la ampliación de las evaluaciones a diversos dominios de competencias, más allá de las áreas inicialmente cubiertas por PISA, necesarias para garantizar el bienestar personal, social y económico (OCDE, 2005). Como es sabido, el término competencia procede originalmente del ámbito laboral, en el que las competencias se orientan a la realización de tareas eficaces relacionadas con una ocupación o desempeño profesional claramente definido, e implican la puesta en práctica de un conjunto de conocimientos, habilidades y actitudes (Zabala \& Arnau, 2007, p. 37). Su recepción en la campo de la educación recoge los planteamientos del mundo laboral, pero amplía su significado. Así, la OCDE, en el marco del proyecto DeSeCo, no dudó en señalar que "una competencia es más que conocimientos y destrezas. Involucra la habilidad de enfrentar demandas complejas, apoyándose en y movilizando recursos psicosociales (incluyendo destrezas y actitudes) en un contexto en particular" (OCDE, 2005, p. 3). Dentro de los recursos que supone la acción competente, tan importantes como las destrezas son los valores y la capacidad de actuar reflexivamente, que "involucra no sólo la habilidad de aplicar de forma rutinaria una fórmula o método para confrontar una situación, sino también la capacidad de adaptarse al cambio, aprender de las experiencias y pensar y actuar con actitud crítica" (ibid., p. 4).

Nuestro objetivo aquí será mostrar qué posibilidades de filiación existen entre este enfoque del trabajo pedagógico y las propuestas de John Dewey. Su propia conceptuación de la educación como reconstrucción continua de la experiencia (Dewey, 1916/1995, pp. 73-76), sugiere algún tipo de vinculación con el concepto de competencia tal como lo formuló, por ejemplo, el proyecto DeSeCo. Otros aspectos de su filosofía de la educación nos mueven, sin embargo, en la dirección opuesta. Él no dedicó ningún análisis específico al término con las connotaciones actuales. Pretender tal cosa sería un anacronismo. La palabra aparece dispersa a lo largo de su ingente obra en algunas pocas ocasiones, a menudo con un significado genérico. Más sugerente es el hecho de que nunca propusiese una lista cerrada de algo parecido a los actuales catálogos de competencias que orientase el trabajo la educación. Ahora bien, podría argumentarse que lo que justifica apelar a Dewey como antecedente del enfoque no es tanto su pedagogía explícita como la base filosófica que la sustenta. Frente a esta hipótesis, intentaremos mostrar que el rechazo a un catálogo de competencias, tan al uso hoy, se explica desde la propia filosofía, y más específicamente la filosofía política, de Dewey tal como él la expuso en The Public and its Problems (1927) e Individualism, Old and New (1930) obras de eminente carácter sociológico y político que apenas son tenidas en cuenta a la hora de analizar su contribución pedagógica. 
Desarrollaremos el argumento en tres fases. En primer lugar, rastrearemos las posibilidades de filiación del aprendizaje por competencias en el marco del empeño deweyano de reconstrucción democrática, mediante la exhortación a un nuevo individualismo que lograse conciliar el desarrollo de la individualidad con la participación en un espíritu de vida comunitario. A continuación, llevaremos el análisis al rechazo de Dewey a los dualismos y los debates que mantuvo con respecto a la articulación de la formación profesional y la educación general. Por último, nos ocuparemos de las consecuencias que para el enfoque de las competencias cabe extraer de la propuesta de cambio de una lógica absolutista a una lógica de la experimentación, que caracterizó la tarea filosófica de Dewey.

\section{Las competenciasen en el marco de la búsqueda de un nuevo individualismo}

Individualism, Old and New recoge una serie de artículos publicados por Dewey en los meses previos e inmediatamente posteriores al crack de 1929. Como ha señalado Morán (2009) el punto de arranque de Dewey es el conflicto que en ese momento asolaba a la sociedad norteamericana, y que para él tenía su origen en la devastadora contradicción entre las creencias y teorías que cimentaban esta sociedad, por un lado, y el modo en que las mismas estaban siendo puestas en práctica, por otro. El efecto de esta contradicción era una inclinación cada vez más ostensible hacia el culto al dinero y la exaltación del éxito económico, de modo que los viejos ideales de libertad, igualdad y desarrollo de una auténtica individualidad, que siempre habían formado parte de la tradición y el legado norteamericanos, se mostraban como irreconocibles:

"El factor espiritual de nuestra tradición, la
igualdad de oportunidades y la libre asociación
e intercomunicación, se ha visto oscurecido y
desplazado. En lugar del desarrollo de aquellas
individualidades que profetizaba, lo que se da es
una perversión del ideal entero de individualismo
para ajustarse a las costumbres de una cultura
del dinero. Se ha convertido en la causa y la
justificación de las desigualdades y opresiones.
De ahí nuestros compromisos, así como los
conflictos en los que los fines y criterios se han
distorsionado hasta lo irreconocible" (Dewey,
1930a/2003, p. 60).

El prototipo de individuo americano que se imponía por aquel entonces en los Estados Unidos, y había empezado a expandirse por otros países, se caracterizaba por su carencia de una verdadera personalidad, esto es, por su despersonalización, estandarización y uniformidad. Estos eran para Dewey los rasgos más representativos de ese individuo engendrado en el seno del "capitalismo corporativo" que entonces se estaba desarrollando. El mencionado crack del 29 y la Gran Depresión de los años treinta supusieron la quiebra del optimismo y la confianza en el futuro que había surgido al amparo del anterior clima de prosperidad, e hirieron de muerte a ese otro componente del sueño americano del "hombre hecho a sí mismo". Durante el tan proclamado y celebrado "reino del individualismo", lo que realmente estaba teniendo lugar era el ocaso y declive de la auténtica individualidad, tal como expresa Morán a propósito de Dewey:

\footnotetext{
"La persistencia de este individualismo 'económico egoísta', como lo denomina el propio Dewey, guiado exclusivamente por el beneficio económico privado y cuyos criterios de valor predominantes provendrían del éxito monetario, se erigiría así en el principal obstáculo para la creación de un nuevo tipo de individuo cuyos esquemas de pensamiento y acción vinieran permanentemente marcados por el consenso, la sociabilidad y la cooperación con los demás. De suerte que la autocomplaciente y narcisista proclamación del 'americanismo' como arquetipo ejemplar de individualismo no dejaba de ser, a fin de cuentas, más que un símbolo de la creciente cuantificación, estandarización e indiferenciación que se iba adueñando progresivamente de la vida de la sociedad norteamericana" (Morán, 2009, p. 20).
} 
Ante esta situación, para Dewey la supervivencia de la democracia americana requerirá impulsar una cultura individualista de nuevo cuño, capaz de hacer suyos los valores de la energía creativa y la iniciativa individual bajo las nuevas condiciones sociales abiertas por la ciencia y la tecnología. Para ello Dewey, en abierto contraste con la teoría económica del laissez-faire, defiende la necesidad de establecer una cierta planificación o control público de la industria y la economía, acometiendo cambios más profundos y radicales que los impulsados por las propuestas reformistas del New Deal. Frente al "viejo liberalismo", Dewey propone, así, un "nuevo liberalismo" o, como él lo denomina también, "socialismo público", capaz de combinar la defensa de la libertad personal característica del liberalismo clásico con una mayor igualdad social:

"El determinismo económico es un hecho, no
una teoría. Pero hay una diferencia y una opción
entre elegir un determinismo ciego, caótico y
carente de planificación, fruto de una economía
que sólo persigue el beneficio pecuniario, o la
determinación de un desarrollo socialmente
planificado y ordenado. La diferencia y la elección
entre un socialismo capitalista y un socialismo
público" (Dewey, 1930a/2003, p. 137).

Puesta en este marco, la filosofía política de Dewey resulta difícilmente armonizable con un concepto de competencia directamente relacionado con las demandas del mercado, en el que se ha visto una encarnación del tipo de individualismo económico egoísta que Dewey deploró en su época (Benade, 2012, pp. 209-211). Por ello, no hay que extrañarse que Martha Nussbaum, erigida en alternativa al enfoque de las competencias (Lozano et al., 2012), invoque precisamente, a Dewey para defender una educación orientada por valores distintos a los del beneficio económico, si bien, contrariamente a lo que él postuló, suprimiendo el papel del control social en su promoción (Mougan, 2013). Nussbaum acude, entre otros, al filósofo americano para recordar la tradición pedagógica que acentúa la participación activa de los alumnos en el proceso de aprendizaje, y que "se resiste con obstinación al modelo basado en el crecimiento económico" (Nussbaum, 2010, p. 39). Lo que ella encuentra hoy como orientación prioritaria en el mundo de la educación es algo muy diferente a lo que se reclama desde esa tradición, y relata el siguiente episodio sucedido en la escuela que dirigió Dewey, cien años más tarde de su marcha:

\begin{abstract}
"En noviembre de 2005, se organizó un retiro para docentes en la Laboratory School de Chicago, una institución que se encuentra en el predio de mi propia universidad y donde John Dewey puso en práctica sus experiencias para la reforma democrática del sistema educativo, la misma institución donde las hijas del presidente Barack Obama pasaron los primeros años de su formación. Los docentes se reunieron a fin de debatir sobre la educación para la ciudadanía democrática y analizaron una gran variedad de experiencias educativas, estudiando a figuras de la tradición occidental, como Sócrates y Dewey, y de la misma línea en la tradición oriental, como Tagore en la India. Sin embargo, se advirtió que algo estaba fallando. Los docentes, que se enorgullecen de estimular a sus alumnos para que cuestionen, critiquen y utilicen la imaginación, expresaron su preocupación por las presiones recibidas de las familias pudientes que envían a sus hijos a esta escuela de élite. Impacientes con los aprendizajes que consideran superfluos y ansiosos de que sus hijos adquieran aptitudes comprobables y tendentes al éxito económico, estos padres están tratando de cambiar los principios rectores de la escuela. $Y$, al parecer, están listos para lograrlo" (ibid., pp. 22-23).
\end{abstract}

Nussbaum aboga por contrarrestar la tendencia actual a priorizar los objetivos económicos de la educación, que patentiza este episodio, reforzando un cultivo de las humanidades que recupere el valor del pensamiento crítico, la imaginación creativa y la capacidad empática, como requisitos "para mantener a la democracia con vida y en estado de alerta" (ibid., p. 29), esto es, como vía para relanzar la democracia entendida como una forma de vida que reivindicó Dewey. 


\section{El rechazo a los dualismos}

La situación paradójica con la que nos encontramos es, pues, ver que a Dewey se recurre no solo como antecedente del enfoque de las competencias, sino también como referente de una posible alternativa al mismo. La pedagogía de Dewey puede, de hecho, leerse como el intento de ir más allá del posible dualismo entre estas dos opciones. Esto no significa que desde esta pedagogía todo valga. Por el contrario, como veremos, desde la misma emergen exigencias muy concretas para un enfoque de las competencias que quiera tener a Dewey por su valedor.

Como señaló Westbrook, para Dewey muchos problemas de la práctica educativa de su época se debían a que estaban sustentados en dualismos erróneos que oponen mente y mundo, pensamiento y acción, teoría y práctica (Westbrook, 1993). El intento de vencer estos dualismos, enfrentó a Dewey tanto con los partidarios de una educación tradicional "centrada en el programa" como con los reformadores que abogaban por una educación "centrada en el niño". Para los tradicionalistas, representados por el neohegeliano William Torrey Harris, el contenido de la instrucción era la cuestión prioritaria a tener en cuenta por el profesor. El currículo debía responder, no a los caprichosos intereses del niño, sino al saber acumulado por la civilización, que Harris concentraba en "las cinco ventanas del alma": aritmética y matemáticas, geografía, historia, gramática y literatura y arte (Kliebard, 2004, pp. 30-36). En School and Society, publicado originalmente en 1899, Dewey criticaba duramente esta concepción de la educación, y proponía darle un giro copernicano, "trasladando el centro de gravedad de la tierra al sol. En este caso el niño se convierte en el sol, hacia el cual se vuelven todos los instrumentos de la educación; él es el centro con respecto al cual se organizan" (Dewey, 1899/s.f., p. 56).

Pero Dewey -y esto es algo que no siempre se recuerda- fue igualmente crítico, especialmente su etapa madura, con la educación centrada en el niño (child-centered school), bandera del movimiento de educación progresiva, planteada como antítesis al valor del contenido de la enseñanza. En su ensayo How Much Freedom in New Schools? de 1930, mantuvo que las interpretaciones más habituales de la educación centrada en el alumno caían en el mismo error que trataban de evitar:

\section{"Están todavía obsesionadas por el factor personal, no conciben ninguna alternativa a la dirección de los adultos. Lo que se requiere es alejarse de cualquier modo de dirección personal y forma de mero control personal. Cuando el énfasis se pone en tener experiencias que son educativamente valiosas, el centro de gravedad se desplaza del factor personal, para situarse dentro de una experiencia en desarrollo en la que alumnos y profesores participan" (Dewey, 1930c/2008, p. 322).}

En el fondo, bajo una y otra crítica, lo que hay es un rechazo a una educación fundada en la autoridad que da al educador la presunción de posesión de un conocimiento inmutable, a la que Dewey opone una educación que encarne en sus propios principios y métodos la idea de la democracia como "experiencia comunicada conjuntamente" (Dewey, 1916/1995, p. 82).

El mismo rechazo a los dualismos y a una educación establecida sobre un principio de predestinación social, es lo que llevó a Dewey a oponerse tanto a una educación profesional anti-intelectual y acientífica, limitada al entrenamiento en destrezas mecánicas, como a una educación liberal ajena a las condiciones de la vida del momento, tal como reconoce Nussbaum (Nussbaum, 2010, pp. 121-122). El rechazo de Dewey a ambas formas de entender el trabajo educativo quedó también patente en dos conocidas polémicas que mantuvo a lo largo de su vida académica. La primera de ellas fue la que le enfrentó con David Snedden, entonces Comisario de Educación de Massachusetts, hacia 1914 y 1915, mientras preparaba Democracia y educación (Labaree, 2010). Snedden defendía los beneficios de un sistema de educación vocacional o profesional, separado de una educación liberal, que preparase a las bases 
sociales para ser productores eficientes. Dewey, que mantuvo siempre la necesidad de pensar unidas la educación profesional y la general, cuya separación era para él vestigio de formas superadas de organización social (Dewey, 1913) criticó duramente el eficientísimo social de Snedden:

\begin{abstract}
"Yo iría más lejos de lo que él parece dispuesto a ir cuando sostiene que la educación debe ser profesional, pero en nombre de una educación verdaderamente profesional, me opongo a la identificación de la actividad laboral con las operaciones que se pueden aprender, digamos, antes de la edad de dieciocho o veinte años, y con la identificación de la educación con la adquisición de habilidades especializadas en el manejo de máquinas, a expensas de una inteligencia industrial basada en la ciencia y el conocimiento de los problemas y las condiciones sociales. Me opongo a considerar como educación profesional ninguna formación que no tenga como su suprema inquietud el desarrollo de dicha iniciativa inteligente, el ingenio y la capacidad ejecutiva que hagan de los trabajadores, hasta el máximo posible, los señores de su propio destino industrial. Tengo mis dudas acerca de la predestinación teológica, así como de todo aquello en lo que el dogma asigna poder predestinador a un ser omnisciente; y soy totalmente opuesto a dar el poder de predestinación social, por medio de una capacitación profesional estrecha, a cualquier grupo de hombres falibles no importa lo bien intencionados que sean" (Dewey, 1915/1979, p. 411).
\end{abstract}

En Democracia y educación Dewey mantendrá, así, la importancia de que en la sociedad democrática todos los ciudadanos dispongan de una competencia industrial o profesional que les capacite para ganarse la vida y no ser una rémora social, pero alertará sobre el peligro de que al insistir sobre este fin se acepten como definitivas las condiciones y normas económicas existentes (Dewey 1916/1995, p. 107). En consecuencia, considerará que no se puede restringir la competencia profesional a un domino técnico que perpetúe el orden social dado:

\footnotetext{
"La competencia técnica falta con frecuencia desgraciadamente, y sería de desear en todos sentidos, no sólo por la producción de mejores artículos a menos costo, sino por la mayor felicidad que se encuentra en el trabajo. Pues nadie
}

se preocupa de lo que no puede hacer bien. Pero hay una gran diferencia entre una competencia limitada al trabajo inmediato y una competencia extendida a una visión de sus efectos sociales, entre la eficiencia para realizar los planes de otros y la de formar los propios" (ibid., p. 267).

La segunda polémica fue la que veinte años más tarde sostuvo Dewey con Robert M. Hutchins - entonces Presidente de la Universidad de Chicago, en la que Dewey había trabajado hasta 1904- con ocasión de la publicación del libro The Higher Learning in America (1936), fruto de los planes de reforma que Hutchins pretendió llevar a cabo animado por Mortimer J. Adler (Jover \& Gozálvez, 2012). En el libro, Hutchins hace un análisis del desconcierto reinante en los estudios universitarios, del que culpa al afán por ganar dinero, que lleva a confundir sus fines, cierto sentido incorrecto de la democracia, para el cual todos tienen que tener acceso al mismo tipo y grado de educación, y una noción errónea del progreso, que niega el pasado y enaltece el conocimiento empírico. La combinación de estos tres factores origina el predomino de la función profesionalizadora, la cual priva a la universidad de "el único pretexto para su existencia, que es proporcionar un refugio en donde la búsqueda de la verdad pueda continuar sin trabas más allá de la utilidad o la presión por los resultados" (Hutchins, 1936, p. 43).

Dewey compartía algunos aspectos del diagnóstico de la situación hecho por Hutchins, pero no podía asumir su planteamiento del problema, que para él se basaba en dos premisas inaceptables acerca del conocimiento: a) la creencia en la existencia de principios y verdades fijas y eternas y b) la necesidad de distanciar lo más posible la educación superior de la vida social contemporánea. Dewey llega a caracterizar esta concepción del conocimiento de autoritaria y presa fácil de posiciones fascistas:

\footnotetext{
"Cualquier esquema que se base en la existencia de primeros principios fundamentales, con su dependiente jerarquía de principios subsidiarios, no escapa del autoritarismo al considerar tales principios como 'verdades'. No quisiera insinuar que el autor tiene alguna simpatía por el fascismo. Pero básicamente su idea de cómo ha de ser asumido el curso apropiado de las
} 
cosas es afín a una desconfianza en la libertad y a la correspondiente apelación a cierta autoridad fija que ahora está invadiendo el mundo. Está implícita en cualquier afirmación de primeras verdades eternas y fijas la necesidad de admitir alguna autoridad humana que decida, en este mundo conflictivo, cuáles son precisamente estas verdades y cómo deben ser enseñadas" (Dewey, 1937a, p. 104).

Para Dewey, la respuesta a la confusión de la universidad no podía buscarse en una concepción del conocimiento que ya no responde a la situación de la sociedad actual. La única alternativa posible es la que abre el conocimiento basado en la experiencia:

"Propongo una alternativa, aunque creo que no hay
una alternativa última; a saber, el lugar primario
de la experiencia, del método experimental,
y de la conexión integral con la práctica en la
determinación del conocimiento, y el papel auxiliar
de lo que se denomina Razón e Intelecto en la
tradición clásica" (Dewey, 1937b, p. 167).

\section{Las competencias en la lógica de la experimentación}

Dos conclusiones cabe extraer para el tema que nos ocupa de los debates pedagógicos en los que se embarcó Dewey en su rechazo a los dualismos. La primera es la necesidad de una mayor articulación entre una preparación profesional, atenta a las circunstancias y necesidades sociales, y una formación general o liberal enfocada a la acción con sentido. La segunda, derivada de la misma concepción del conocimiento, es la de la sustitución de la vieja lógica autoritaria o absolutista, por una lógica de la experimentación. Si la primera conclusión puede servir de apoyo a las actuales políticas del aprendizaje por competencias, la segunda indica el modo de su realización a partir de la pedagogía de Dewey, realización que, como pretendemos mostrar en este último apartado, entra en contradicción con las prácticas que impulsan esas políticas, las cuales se obstinan en prefijar el perfil de persona y profesional competente, mediante el establecimiento de catálogos interminables de competencias que anticipan los resultados de aprendizaje a lograr.

La dicotomía entre absolutismo y experimentalismo fue tan potente en Dewey que eligió esta fórmula como título de un texto autobiográfico de finales de los años veinte para expresar su propia trayectoria filosófica (Dewey, 1930b/2008). En Theory of Valuation, obra de carácter eminentemente epistemológico publicada en 1939 en la International Encyclopedia of Unified Sciences (Dewey, 1939/2008) abordó esta distinción a propósito de la relación entre medios y fines, como una forma de situarse en la polémica, auspiciada por el auge del positivismo lógico, acerca de la separación entre cuestiones de hecho y juicios de valor. Frente al absolutismo de los "fines-en-sí-mismos" como criterio último de toda valoración, Dewey sostuvo la continuidad fundamental entre medios, fines, deseos y preferencias, cuya validez reposa en su carácter instrumental en el progreso de la experiencia humana. En este sentido, hechos y valores dejan de ser dos realidades dicotómicas para emerger como una única cuestión a la que solo se puede tener acceso desde "la valoración", es decir, desde la actividad de participación en una comunidad de conocimiento.

Poco antes, Dewey había abordado esta confrontación desde el ángulo de la filosofía política en The Public and its Problems. El libro es una reacción a las tesis del periodista y comentarista político Walter Lippmann con respecto al sentido que convendría dar a la ciudadanía en el contexto de las democracias liberales. En él, Dewey se refirió también al concepto de "competencia", en esta ocasión bajo la forma de la teoría del ciudadano "omnicompetente" que postuló Lippmann como mito del gobierno democrático. La referencia se produce en el contexto de la búsqueda de las condiciones de constitución de una Gran Comunidad genuinamente democrática que permita a lo público emerger de su eclipse. Para ello, Dewey comienza refiriéndose a la imposibilidad de seguir manteniendo cierto sistema habitual de creencias sobre la configuración del Estado democrático, que los acontecimientos habrían 
demostrado ser erróneo. Escribe:

"En la base del sistema yace lo que Lippmann ha denominado con acierto la idea del individuo 'omnicompetente': competente para diseñar planes políticos, para juzgar sus resultados; competente para saber, en todas las situaciones que exijan una acción política, qué conviene para su propio bien; y competente para hacer realidad su idea de bien y de voluntad de llevarla a la práctica contra fuerzas opuestas. La historia posterior, sin embargo, ha demostrado que esta suposición implicaba una ilusión" (Dewey, 1927/2004, pp. 142-143).

Para Dewey la disfunción de las democracias liberales provendría de la falsedad de este sistema de creencias, que ha logrado mantenerse "por la engañosa influencia de una falsa psicología", para la cual "las ideas y el conocimiento eran funciones de una mente o una conciencia que se originaban en los individuos por medio de un contacto aislado con los objetos" (ibid., p. 143). La democracia liberal no se funda, como sostiene Lippmann, en el mito de la omnicompetencia de sus ciudadanos, sino en la participación de los individuos en la reglamentación de las formas de su propia existencia, tal y como ha destacado Zask interpretando a Dewey (Zask, 2008, p. 170). Para esta autora, el paso de una concepción a la otra, marca el tránsito de la lógica del absolutismo a la lógica de la experimentación, que para ella constituye la principal aportación de la filosofía política de Dewey (ibid., p. 186).

El propio Dewey se encargó de mostrar las implicaciones de este tránsito para la educación. La lógica absolutista se orienta por una imagen preconcebida de los fines a lograr. "Incluso cuando los procesos de la educación no se proponen la perpetuación inmutable de las instituciones existentes, se supone que debe tenerse una imagen mental de algún objetivo deseado, personal y social que se debe alcanzar, y que esa concepción de un fin fijo y determinado debería controlar los procesos educativos. Los reformistas comparten con los conservadores esta convicción" (Dewey, 1927/2004, pp. 164-165). La lógica de la experimentación se mueve en otra dirección. En ella, los conceptos y las teorías con que se forma el conocimiento se configuran como "herramientas de investigación", y las propuestas de acción se tratan "como hipótesis de trabajo, no como programas que deban seguirse y ejecutarse de forma rígida" (ibid., p. 166).

En Dewey, la formación en competencias debe subsumirse necesariamente en una metodología de experimentación que aproxima, como ha señalado Zask, la lógica de su desarrollo a la de la obtención de un acuerdo (Zask, 2008, p. 179). Es decir, bajo la égida de la lógica de la experimentación que propone Dewey, el currículo no se articula en unos contenidos que transmita el profesor y unos objetivos preestablecidos que el alumno deba alcanzar a través de la adquisición de una serie determinada de competencias, tal y como las conocemos hoy, sino que el aprendizaje se realiza a través de la superación de los problemas propuestos por el profesor, que implica una actividad de discusión y acuerdo con los compañeros que configuran la comunidad de conocimiento del aula, en la que cada cual participa democráticamente aportando todos los rasgos de su individualidad (carácter, habilidades y virtudes; lo que hoy llamaríamos conocimientos, habilidades $\mathrm{y}$ talentos propios). Cobra, así, pleno sentido la apelación a Dewey que propone Nussbaum en su rechazo al individualismo egoísta que fomenta la educación actual. La autora subraya especialmente en Dewey el uso pedagógico de la mayéutica socrática. Pero no se trata solo de una técnica de aprendizaje, sino de la inmersión en una situación de construcción de vida en común:

"Según Dewey, la mejor manera de lograr que los alumnos fueran personas activas era transformar al aula en un espacio del universo real, que guardara continuidad con el mundo exterior, un espacio donde se debatieran problemas de la vida misma y se recurriera a las aptitudes prácticas más concretas. Por lo tanto, el elemento socrático no se limitaba a las capacidades intelectuales, sino que constituía un aspecto de las actividades prácticas: era toda una postura frente a los problemas de la vida real. Asimismo, suponía también una forma de interactuar con los demás. Dewey siempre sostuvo que en una buena escuela, los alumnos aprenden a ser ciudadanos realizando proyectos en común con sus compañeros y resolviéndolos en conjunto, con un espíritu respetuoso pero, al mismo tiempo, crítico. De acuerdo con este autor, 
la actividad cooperativa conllevaba la ventaja agregada de impartir respeto por las labores manuales y por los oficios, mientras que las escuelas convencionales en general mostraban cierta preferencia elitista por las ocupaciones sedentarias. Por lo tanto, el socratismo en Dewey no se limitaba a aplicar una técnica por la que los alumnos aprendieran a argumentar sentados en sus pupitres, sino que se trataba de una forma de vida compartida con los demás en la búsqueda de respuestas para cuestiones concretas del mundo real y la realización de proyectos prácticos con la guía de los maestros, pero sin imposición de autoridad externa alguna" (Nussbaum, 2010, p. 97).

Pasar de una lógica absolutista a una lógica de la experimentación, significa que los alumnos no deben ser tratados como seres preconstituidos por el dominio potencial de una serie de competencias que la escuela deba hacer aflorar, sino como seres libres que crecen y desarrollan su libertad mediante la investigación, la experimentación y la discusión compartida con sus compañeros en el aula. Cabe, por ello, afirmar que mientras que la actual propuesta de aprendizaje por competencias es compatible con la libertad positiva (entendida como "libertad para" en el sentido de libertad de acción) al menos desde un punto de vista teórico, en la medida en que llegue a promover el aprendizaje a través de la resolución de problemas

\section{Conclusión}

La necesidad de adaptar los sistemas de educación y formación a las necesidades planteadas por la sociedad y la economía actual, ha hecho que el aprendizaje por competencias se haya instalado con fuerza en el discurso pedagógico y las políticas educativas nacionales e internacionales. En consecuencia, tiene sentido preguntarse por la fundamentación teórica de este concepto, que orienta hoy el mundo de la educación. Este ha sido uno de los aspectos que han impulsado el creciente interés por el pragmatismo y por John Dewey, al que se ha identificado como antecedente del aprendizaje por competencias. Si bien es cierto que los planteamientos del conectados con la vida diaria, frente a la asimilación de contenidos transmitidos por la autoridad del profesor, los planteamientos de Dewey van más lejos y apoyan un currículo que promueva tanto la libertad positiva como la libertad negativa del alumnado (entendida como "libertad de" en el sentido de ausencia de un programa cerrado anticipatorio de los resultados a lograr) aunque él no lo dijese con estas palabras.

El compromiso de Dewey con la transformación social del momento y la crítica al liberalismo económico que imperaba en su época en América, fue tal que no se conformó con postular la libertad de agencia, de acción del alumnado como metodología a utilizar dentro del aula. Su apuesta por la libertad individual en su sentido más radical, le impedía formular catálogos cerrados de competencias que prefijasen el ideal de persona formada. Así como los ciudadanos no son seres predefinidos por su omnicompetencia en las variadas dimensiones de la acción política, sino que se desarrollan como tales en el ejercicio de su libertad y participación democráticas, el alumno en la pedagogía de Dewey posee libertad para resolver a través de su propia individualidad y creatividad las tareas a las que se enfrenta, y crece educativamente en la propia experiencia de su resolución compartida.

filósofo y pedagogo americano en torno al aprendizaje a través de la acción muestran cierta relación con los planteamientos recogidos en los documentos elaborados por las instituciones internacionales en torno al concepto de competencia, existen también aspectos que impiden hablar de una relación lineal.

Como hemos tratado de mostrar a lo largo del presente trabajo, por importante que fuera para Dewey la escuela como un ámbito de experimentación de la psicología funcional y el pragmatismo, todavía fue más importante como expresión de su concepción política. La escuela para Dewey 
fue ante todo un laboratorio en el que los alumnos debían desarrollar los hábitos de participación en una vida democrática. Tal era el compromiso de Dewey con su ideal de democracia, que desarrolló todo un sistema filosófico que pretendía sentar las bases de una educación que diera paso a una nueva forma de comunidad, en un momento en el que la sociedad americana se desintegraba. Para ello, no dudó en poner en relación la filosofía con la educación y la política, el pragmatismo epistemológico con el ejercicio de la democracia, a través de los planteamientos de un aprendizaje en acción que adopta la

\section{Referencias}

Benade, L. (2012). Ethical Teaching in the Context of Globalised Education Reform. London: Continuum.

Dewey, J. (1899/s.f.). La escuela y la sociedad. Madrid,: Francisco Beltrán.

Dewey, J. (1913). Liberal Education, en: Monroe, P. (Ed.) A Cyclopedia of Education. New York, Macmillan Co., vol. 4, pp. 4-6.

Dewey, J. (1915/1979). Education versus Trade-Training: Reply to David Snedden, en Boydston, J.A. (Ed.) The Collected Works of John Dewey. The Middle Works, 1899-1924, Volume 8: 1915. Carbondale y Edwardsville: Southern Illinois University Press, pp. 411413.

Dewey, J. (1916/1995). Democracia y educación. Madrid: Morata.

Dewey, J. (1927/2004). La opinión pública y sus problemas. Madrid: Morata.

Dewey, J. (1930a/2003). Viejo y nuevo individualismo. Barcelona: Paidós.

Dewey, J. (1930b/2008). From Absolutism to Experimentalism, en Boydston, J.A. (Ed.) The Collected Works of John Dewey. The Later Works, 1925-1953, Volume 5: 19291930. Carbondale y Edwardsville: Southern forma de una investigación en comunidad.

La filosofía de la educación que emana de este compromiso, no necesariamente es contraria a una formación en competencias, si por ello se entiende una educación basada en el aprendizaje a través de la acción y la experimentación que esté atenta a las necesidades sociales del momento. Pero Dewey habría sido reacio a una formación basada en una serie cerrada de competencias, que prefijase un determinado perfil de la persona a alcanzar, obstruyendo la libertad y la creatividad del alumno.

Illinois University Press, pp. 147-162.

Dewey, J. (1930c/2008). How Much Freedom in New Schools? en Boydston, J.A. (Ed.) The Collected Works of John Dewey. The Later Works, 1925-1953, Volume 5: 1929-1930. Carbondale y Edwardsville: Southern Illinois University Press, pp. 319-325.

Dewey, J. (1937a). John Dewey's Page: President Hutchins' Proposals to Remake Higher Education. Social Frontier, 22 (3), pp. 103-104.

Dewey, J. (1937b). The Higher Learning in America. Social Frontier, 24 (3), pp. 167-169.

Dewey, J. (1939/2008). Teoría de la Valoración. Madrid: Siruela.

Giles, D. E. \& Eyler, J. (1994). The theoretical roots of service learning in John Dewey: Towards a theory of service learning. Michigan Journal of Community Service Learning, 1, pp. 77-85.

Hutchins, R.M. (1936). The Higher Learning in America. New Brunswick: Transaction Pub.

Jover, G. \& Gonzálvez, V. (2012). La universidad como espacio público: un análisis a partir de dos debates en torno al pragmatismo. Bordón, Revista de Pedagogía, 
64 (3), pp. 39-52.

Kliebard, H. M. (2004). The Struggle for the American Curriculum, 1893-1958. New York: RoutledgeFalmer.

Koschmann, T. (2000). The Physiological and the Social in the Psychologies of Dewey and Thorndike: The Matter of Habit, en Fishman B. y O'connor-Divelbiss, S. (Eds.) Fourth International Conference of the Learning Sciences. Mahwah: Erlbaum, pp. 314-319. Recuperado de:

http://www.umich.edu/ icls/proceedings/ pdf/Koschmann.pdf

Labaree, D.F. (2010). How Dewey Lost: The Victory of David Snedden and Social Efficiency in the Reform of American Education, en Trohler, D; Schlag, T. y Osterwalder, F. (Eds.) Pragmatism and Modernities. Rotterdam: Sense Publishers, pp. 163-188.

Lozano, J. F., Boni, A., Peris, J. \& Hueso, A. (2012). Competencies in Higher Education: A Critical Analysis from the Capabilities Approach. Journal of Philosophy of Education, 46 (1), pp. 132-147.

http://dx.doi.org/10.1111/j.1467-

9752.2011.00839.x

McCowan, R.J. (1998). Origins of Competency-Based Training. Buffalo: Center for Development of Human Services. Recuperado de

http://files.eric.ed.gov/fulltext/ED501710. pdf

Morán, J. G. (2009). John Dewey, individualismo y democracia. Foro Interno, 9, pp.11-42.
Mougan, J.C. (2013). M. Nussbaum, J. Dewey and Education for Democratic Citizenship. Pragmatism Today, 4 (2), pp. 84-90

Nussbaum, M.C. (2010). Sin fines de lucro. Por qué la democracia necesita de las humanidades. Madrid: Katz.

OCDE (2005). La definición y selección de competencias clave. Resumen ejecutivo Neuchâtel: Federal Statistical Office.

Rodgers, C. (2002). Defining reflection: Another look at John Dewey and reflective thinking. Teachers College Record, 104, pp. 842-866.

http://dx.doi.org/10.1111/1467-9620.00181

Romainville, M. (1996). L'irresistible ascension du terme "compétence" en éducation, Enjeux, 37/38, pp. 132-142.

Westbrook, R.B. (1993). John Dewey (18591952). Perspectivas: revista trimestral de educación comparada, 23 (1-2), pp. 289-305.

You, Z. \& Rud, A.G. (2010). A Model of Dewey's Moral Imagination for Service Learning: Theoretical Explorations and Implications for Practice in Higher Education. Education $\&$ Culture, 26 (2), pp. 36-51.

Zabala, A. \& Arnau, L. (2007). Once ideas clave. Cómo aprender y enseñar competencias. Barcelona: Graó.

Zask, J. (2008). Le public chez Dewey: un e union sociale plurielle. Tracés. Revue de Sciences humaines, 15 (2), pp. 169-189. http://dx.doi.org/10.4000/traces.753 